\title{
HARDY INEQUALITIES FOR A MAGNETIC GRUSHIN OPERATOR WITH AHARONOV-BOHM TYPE MAGNETIC FIELD
}

\author{
L. AERMARK AND A. LAPTEV
}

Dedicated to the memory of M. Sh. Birman whose enormous scientific achievements continue to guide many generations of mathematicians. The strong school in Spectral Theory that he developed is renowned all over the world. All his pupils and colleagues remember Professor Birman as a wonderful person who was always ready to help. His warm and generous support certainly aided the second author of this paper to survive as a mathematician.

\begin{abstract}
A version of the Aharonov-Bohm magnetic field for a Grushin subelliptic operator is introduced; then its quadratic form is shown to satisfy an improved Hardy inequality.
\end{abstract}

\section{$\S 1$ INTRODUCTION AND THE MAIN RESULT}

The classical Hardy inequality states that if $d \geq 3$, then for any smooth function $u$ with compact support we have

$$
\int_{\mathbb{R}^{d}}|\nabla u(x)|^{2} d x \geq\left(\frac{d-2}{2}\right)^{2} \int_{\mathbb{R}^{d}} \frac{|u(x)|^{2}}{|x|^{2}} d x .
$$

By continuity, inequality (11) extends to any function belonging to the homogeneous Sobolev space $\mathcal{H}^{1}\left(\mathbb{R}^{d}\right)$. It is well known that the constant $(d-2)^{2} / 4$ in (1) is sharp but not attained. The literature concerning various versions of Hardy's inequalities and their applications is extensive and we are not able to cover it in this short paper. We only mention the classical paper of M. Sh. Birman [1], the article of E. B. Davies [4], and the book of V. Maz'ya [11.

Among many applications of inequality (1) we would like to mention that, in combination with the Schwarz inequality, it implies

$$
\left(\int_{\mathbb{R}^{d}}|x|^{2}|u(x)|^{2} d x\right)\left(\int_{\mathbb{R}^{d}}|\nabla u(x)|^{2} d x\right) \geq\left(\frac{d-2}{2}\right)^{2}\left(\int_{\mathbb{R}^{d}}|u(x)|^{2} d x\right)^{2} .
$$

This estimate takes a particularly symmetric form if in the second integral on the lefthand side we use the Parseval formula for the Fourier transform $\widehat{u}$ of the function $u$ :

$$
(2 \pi)^{d}\left(\int_{\mathbb{R}^{d}}|x|^{2}|u(x)|^{2} d x\right)\left(\int_{\mathbb{R}^{d}}\left[\left.\xi\right|^{2}|\widehat{u}(\xi)|^{2} d \xi\right) \geq\left(\frac{d-2}{2}\right)^{2}\left(\int_{\mathbb{R}^{d}}|u(x)|^{2} d x\right)^{2} .\right.
$$

This inequality expresses the Heisenberg uncertaintly principle, which states that a nontrivial $L^{2}$-function and its Fourier transform cannot be simultaneously very small near the origin.

Hardy's inequalities were also studied for some subelliptic operators (see, e.g., 6, 7, 2, [3, 5, 12, 9]), in particular, for the sub-Laplacian on the Heisenberg group $\mathbb{H}$. This group is the prime example in noncommutative harmonic analysis, and we refer to [13] for the background material.

2010 Mathematics Subject Classification. Primary 35P15; Secondary 81Q10.

Key words and phrases. Hardy inequalities. 
Let us realize $\mathbb{H}$ as $\mathbb{R}^{3}$ with coordinates $(x, y, t)$ and with the (noncommutative) multiplication $(x, y, t) \circ\left(x^{\prime}, y^{\prime}, t^{\prime}\right)=\left(x+x^{\prime}, y+y^{\prime}, t+t^{\prime}-2\left(x y^{\prime}-y x^{\prime}\right)\right)$. The vector fields

$$
X=\frac{\partial}{\partial x}+2 y \frac{\partial}{\partial t}, \quad Y=\frac{\partial}{\partial y}-2 x \frac{\partial}{\partial t}
$$

are left-invariant and the sub-Laplacian on $\mathbb{H}$ is given by

$$
H=-X^{2}-Y^{2}=-\left(\frac{\partial}{\partial x}+2 y \frac{\partial}{\partial t}\right)^{2}-\left(\frac{\partial}{\partial y}-2 x \frac{\partial}{\partial t}\right)^{2} .
$$

The quadratic form $h$ of the operator $H$ is defined by

$$
h[u]=\int_{\mathbb{R}^{3}}\left(|X u|^{2}+|Y u|^{2}\right) d x d y d t .
$$

Let $z=(x, y),|z|=\sqrt{x^{2}+y^{2}}$; we consider the so-called Kaplan distance function from $(z, t)$ to the origin:

$$
d(z, t)=\left(|z|^{4}+t^{2}\right)^{1 / 4} .
$$

The function $d$ is positive homogeneous in the following sense:

$$
d\left(\lambda z, \lambda^{2} t\right)=\lambda d(z, t), \quad \lambda>0,
$$

and it has a singularity at zero.

The Grushin operator

$$
G=-\Delta_{z}-4|z|^{2} \partial_{t}^{2}
$$

(see [8]) gives another example of a subelliptic operator. Its quadratic form $g$ satisfies

$$
g[u]=\int_{\mathbb{R}^{3}}\left(\left|\nabla_{z} u\right|^{2}+4|z|^{2}\left|\partial_{t} u\right|^{2}\right) d z d t .
$$

For the forms (3) and (5), the following sharp Hardy inequalities were obtained and discussed in detail in [6] and [7]:

$$
h[u]=\int_{\mathbb{R}^{3}}\left(|X u|^{2}+|Y u|^{2}\right) d z d t \geq \int_{\mathbb{R}^{3}} \frac{|z|^{2}}{d^{4}}|u|^{2} d z d t
$$

and

$$
g[u]=\int_{\mathbb{R}^{3}}\left(\left|\nabla_{z} u\right|^{2}+4|z|^{2}\left|\partial_{t} u\right|^{2}\right) d z d t \geq \int_{\mathbb{R}^{3}} \frac{|z|^{2}}{d^{4}}|u|^{2} d z d t .
$$

Inequalities (6) and (17) are related to each other. Indeed, the operator $H$ defined in (2) can be rewritten in the form

$$
H u=-\Delta_{z} u-4|z|^{2} \partial_{t}^{2}-4 \partial_{t} T u=G u-4 \partial_{t} T u,
$$

where $T=y \partial_{x}-x \partial_{y}$. In particular, if $u(z, t)=u(|z|, t)$, then $T u=0$, and inequalities (6) and (7) coincide on this subclass of functions.

The classical Hardy inequality (11) becomes trivial in the two-dimensional case. In [10], it was noticed that for some magnetic forms in dimension two the Hardy inequality is fulfilled in its classical form. For example, if $\beta A$ is the Aharonov-Bohm magnetic field

$$
\beta A=\beta\left(\frac{-y}{x^{2}+y^{2}}, \frac{x}{x^{2}+y^{2}}\right), \quad \beta \in \mathbb{R},
$$

then

$$
\int_{\mathbb{R}^{2}}|(\nabla+i \beta A) u|^{2} d x d y \geq \min _{k \in \mathbb{Z}}|k-\beta|^{2} \int_{\mathbb{R}^{2}} \frac{|u|^{2}}{x^{2}+y^{2}} d x d y
$$


Here the form on the left-hand side is considered on the function class $\mathcal{H}^{1}\left(\mathbb{R}^{2}\right)$, obtained by completion of the class $C_{0}^{\infty}\left(\mathbb{R}^{2} \backslash 0\right)$ with respect to the metric defined by the form

$$
\int_{\mathbb{R}^{2}}\left(|\nabla u|^{2}+|x|^{-2}|u|^{2}\right) d x
$$

The constant $\beta$ can be interpreted naturally as the magnetic flux through a disk centered at the origin.

In this paper we introduce a suitable notion of an Aharonov-Bohm type vector field for the Grushin operator $G$ defined in (44), and we obtain an analog of the Hardy inequality (7) for the corresponding magnetic form.

First, we define the "Grushin vector field" as

$$
\nabla_{G}=\left(\partial_{x}, \partial_{y}, 2 x \partial_{t}, 2 y \partial_{t}\right)
$$

Clearly,

$$
G=-\left|\nabla_{G}\right|^{2}
$$

Now we introduce an Aharonov-Bohm type magnetic field:

$$
\mathcal{A}=\left(\mathcal{A}_{1}, \mathcal{A}_{2}, \mathcal{A}_{3}, \mathcal{A}_{4}\right)=\left(-\frac{\partial_{y} d}{d}, \frac{\partial_{x} d}{d},-2 y \frac{\partial_{t} d}{d}, 2 x \frac{\partial_{t} d}{d}\right) .
$$

Then the magnetic Grushin operator with the magnetic field $\beta \mathcal{A}$ and with the "flux" $\beta \in \mathbb{R}$ can be defined as

$$
G_{\mathcal{A}}=-\left(\nabla_{G}+i \beta \mathcal{A}\right)^{2}
$$

Our main result is the following theorem.

Theorem 1. Assume that $-1 / 2 \leq \beta \leq 1 / 2$. Then, for the quadratic form of the magnetic Grushin operator (9), we have the following Hardy inequality:

$$
\int_{\mathbb{R}^{3}}\left(\left|\left(\nabla_{G_{0}}+i \beta \mathcal{A}\right) u\right|^{2} d z d t \geq\left(1+\beta^{2}\right) \int_{\mathbb{R}^{3}} \frac{|z|^{2}}{d^{4}}|u|^{2} d z d t .\right.
$$

Concluding the Introduction, we would like to make some remarks concerning open questions related to subelliptic operators.

Remark 1. It would be interesting to prove a similar result for the Heisenberg quadratic form. It is not clear for us what would be a suitable version of the Aharonov-Bohm magnetic field for this case.

Remark 2. To the best of our knowledge, the notions of the Grushin and Heisenberg Laplacians with constant magnetic fields has not been introduced up to now. It would be interesting to define such operators and to study their spectrum, possibly identifying the notion of the Landau-type levels.

Remark 3. For a multidimensional harmonic oscillator, we have the natural creation and annihilation operators. It would be interesting to define the "harmonic oscillators" with the Heisenberg and Grushin operators and, respectively, creation and annihilation operators related to them.

\section{$\S 2$. Simple proofs of Hardy's inequalities FOR HEISENBERG AND GRUSHIN OPERATORS}

For the sake of completeness, here we present simple proofs of inequalities (6) and (17).

Proposition 1. For any function $u$ with $h[u]<\infty$, the following inequality holds true:

$$
\int_{\mathbb{R}^{3}}\left(|X u|^{2}+|Y u|^{2}\right) d z d t \geq \int_{\mathbb{R}^{3}} \frac{|z|^{2}}{d^{4}}|u|^{2} d z d t .
$$


Proof. It suffices to prove (11) for $u \in C_{0}^{\infty}\left(\mathbb{R}^{3} \backslash 0\right)$. Consider the following nonnegative expression:

$$
I=\int_{\mathbb{R}^{3}}\left|\left(X+\alpha \frac{X d}{d}\right) u\right|^{2} d z d t+\int_{\mathbb{R}^{3}}\left|\left(Y+\alpha \frac{Y d}{d}\right) u\right|^{2} d z d t
$$

where $\alpha \in \mathbb{R}$.

Clearly,

$$
d(z, t)^{-1} X d(z, t)=\frac{x|z|^{2}+y t}{d^{4}(z, t)}, \quad d(z, t)^{-1} Y d(z, t)=\frac{y|z|^{2}-x t}{d^{4}(z, t)} .
$$

Removing the parentheses and integrating by parts, we obtain

$$
\begin{aligned}
I=\int_{\mathbb{R}^{3}}\left(|X u|^{2}+|Y u|^{2}\right) d z d t & -\alpha \int_{\mathbb{R}^{3}}\left(X \frac{X d}{d}+Y \frac{Y d}{d}\right)|u|^{2} d z d t \\
& +\alpha^{2} \int_{\mathbb{R}^{3}}\left(\left(\frac{X d}{d}\right)^{2}+\left(\frac{Y d}{d}\right)^{2}\right)|u|^{2} d z d t \geq 0 .
\end{aligned}
$$

Simple computation gives

$$
\frac{1}{2}\left(X \frac{X d}{d}+Y \frac{Y d}{d}\right)=\left(\frac{X d}{d}\right)^{2}+\left(\frac{Y d}{d}\right)^{2}=\frac{|z|^{2}}{d^{4}},
$$

and we complete the proof by taking $\alpha=1$.

Proposition 2. For any function $u$ such that $g[u]<\infty$, we have

$$
\int_{\mathbb{R}^{3}}\left(\left|\nabla_{z} u\right|^{2}+4|z|^{2}\left|\partial_{t} u\right|^{2}\right) d z d t \geq \int_{\mathbb{R}^{3}} \frac{|z|^{2}}{d^{4}}|u(z, t)|^{2} d z d t .
$$

Proof. Introducing the polar coordinates $x=r \cos \varphi, y=r \sin \varphi, r=|z|$, we obtain

$$
\begin{aligned}
\int_{\mathbb{R}^{3}} & \left(\left|\nabla_{z} u\right|^{2}+4|z|^{2}\left|\partial_{t} u\right|^{2}\right) d z d t \\
& =\int_{-\infty}^{\infty} \int_{0}^{2 \pi} \int_{0}^{\infty}\left(\left|\partial_{r} u\right|^{2}+r^{-2}\left|\partial_{\varphi} u\right|^{2}+4 r^{2}\left|\partial_{t} u\right|^{2}\right) r d r d \varphi d t \\
& \geq \int_{-\infty}^{\infty} \int_{0}^{2 \pi} \int_{0}^{\infty}\left(\left|\partial_{r} u\right|^{2}+4 r^{2}\left|\partial_{t} u\right|^{2}\right) r d r d \varphi d t
\end{aligned}
$$

So, the proof reduces to the inequality

$$
\int_{-\infty}^{\infty} \int_{0}^{\infty}\left(\left|\partial_{r} u\right|^{2}+4 r^{2}\left|\partial_{t} u\right|^{2}\right) r d r d t \geq \int_{-\infty}^{\infty} \int_{0}^{\infty} \frac{r^{2}}{r^{4}+t^{2}}|u|^{2} r d r d t
$$

Let $d=d(r, t)=\left(r^{4}+t^{2}\right)^{1 / 4}$. A simple computation gives

$$
\begin{aligned}
\int_{-\infty}^{\infty} & \int_{0}^{\infty}\left(\left|\left(\partial_{r}+\alpha \frac{\partial_{r} d}{d}\right) u\right|^{2}+4 r^{2}\left|\left(\partial_{t}+\alpha \frac{\partial_{t} d}{d}\right) u\right|^{2}\right) r d r d t \\
= & \int_{-\infty}^{\infty} \int_{0}^{\infty}\left(\left|\partial_{r} u\right|^{2}+4 r^{2}\left|\partial_{t} u\right|^{2}\right) r d r d t \\
& \quad-\int_{-\infty}^{\infty} \int_{0}^{\infty}\left(6 \alpha \frac{r^{2}}{d^{4}}-4 \alpha \frac{r^{6}+r^{2} t^{2}}{d^{8}}-\alpha^{2} \frac{r^{6}+r^{2} t^{2}}{d^{8}}\right)|u|^{2} r d r d t \\
= & \int_{-\infty}^{\infty} \int_{0}^{\infty}\left(\left|\partial_{r} u\right|^{2}+4 r^{2}\left|\partial_{t} u\right|^{2}\right) r d r d t-\int_{-\infty}^{\infty} \int_{0}^{\infty}\left(2 \alpha-\alpha^{2}\right) \frac{r^{2}}{d^{4}}|u|^{2} r d r d t
\end{aligned}
$$

Now, we complete the proof by taking $\alpha=1$. 


\section{§3. Proof of Theorem 1}

Proof. By using the polar coordinates $x=r \cos \varphi, y=r \sin \varphi$ for the $z$-plane, we get

$$
\frac{\partial_{y} d}{d}=\frac{r^{3} \sin \varphi}{r^{4}+t^{2}}, \quad \frac{\partial_{x} d}{d}=\frac{r^{3} \cos \varphi}{r^{4}+t^{2}},
$$

and

$$
2 y \frac{\partial_{t} d}{d}=\frac{y t}{r^{4}+t^{2}}, \quad 2 x \frac{\partial_{t} d}{d}=\frac{x t}{r^{4}+t^{2}} .
$$

Therefore,

$$
\int_{\mathbb{R}^{3}}\left|\left(\nabla_{G_{0}}+i \beta \mathcal{A}\right) u\right|^{2} d z d t=I_{1}+I_{2}
$$

where

$$
\begin{aligned}
I_{1}=\int_{-\infty}^{\infty} \int_{0}^{2 \pi} \int_{0}^{\infty} & \left(\left|\left(\cos \varphi \partial_{r}-\frac{\sin \varphi}{r} \partial_{\varphi}-i \beta \frac{r^{3} \sin \varphi}{r^{4}+t^{2}}\right) u\right|^{2}\right. \\
& \left.+\left|\left(\sin \varphi \partial_{r}+\frac{\cos \varphi}{r} \partial_{\varphi}+i \beta \frac{r^{3} \cos \varphi}{r^{4}+t^{2}}\right) u\right|^{2}\right) r d r d \varphi d t
\end{aligned}
$$

and

$$
\begin{aligned}
I_{2}=\int_{-\infty}^{\infty} \int_{0}^{2 \pi} \int_{0}^{\infty} & \left(\left|\left(2 \cos \varphi r \partial_{t}-i \beta \sin \varphi \frac{r t}{r^{4}+t^{2}}\right) u\right|^{2}\right. \\
+ & \left.\left|\left(2 \sin \varphi r \partial_{t}+i \beta \cos \varphi \frac{r t}{r^{4}+t^{2}}\right) u\right|^{2}\right) r d r d \varphi d t
\end{aligned}
$$

Removing the parentheses, we find

$$
I_{1}=\int_{-\infty}^{\infty} \int_{0}^{2 \pi} \int_{0}^{\infty}\left(\left|\partial_{r} u\right|^{2}+\frac{1}{r^{2}}\left|\partial_{\varphi} u+i \beta \frac{r^{4}}{r^{4}+t^{2}} u\right|^{2}\right) r d r d \varphi d t
$$

We represent $u$ by the Fourier series

$$
u(r, \varphi, t)=\sum_{k=-\infty}^{\infty} u_{k}(r, t) e^{i k \varphi} / \sqrt{2 \pi}
$$

Then, since $-1 / 2 \leq \beta \leq 1 / 2$, we have

$$
\begin{aligned}
\frac{1}{r^{2}} \int_{0}^{2 \pi}\left|\partial_{\varphi} u+i \beta \frac{r^{4}}{r^{4}+t^{2}} u\right|^{2} d \varphi & =\frac{2 \pi}{r^{2}} \sum_{k}\left(k+\beta \frac{r^{4}}{r^{4}+t^{2}}\right)^{2}\left|u_{k}\right|^{2} \\
& \geq \frac{2 \pi}{r^{2}} \min _{k}\left(k+\beta \frac{r^{4}}{r^{4}+t^{2}}\right)^{2} \sum_{k}\left|u_{k}\right|^{2} \\
& =\frac{1}{r^{2}} \min _{k}\left(k+\beta \frac{r^{4}}{r^{4}+t^{2}}\right)^{2} \int_{0}^{2 \pi}|u|^{2} d \varphi \\
& =\beta^{2} \frac{r^{6}}{\left(r^{4}+t^{2}\right)^{2}} \int_{0}^{2 \pi}|u|^{2} d \varphi .
\end{aligned}
$$

Computing $I_{2}$, we arrive at the relation

$$
I_{2}=\int_{-\infty}^{\infty} \int_{0}^{2 \pi} \int_{0}^{\infty} 4 r^{2}\left|\partial_{t} u\right|^{2} r d r d \varphi d t+\int_{-\infty}^{\infty} \int_{0}^{2 \pi} \int_{0}^{\infty} \beta^{2} \frac{r^{2} t^{2}}{\left(r^{4}+t^{2}\right)^{2}}|u|^{2} r d r d \varphi d t
$$


Putting $I_{1}$ and $I_{2}$ together, we conclude that

$$
\begin{aligned}
\int_{\mathbb{R}^{3}}\left|\left(\nabla_{G_{0}}+i \beta \mathcal{A}\right) u\right|^{2} d z d t & \geq \int_{-\infty}^{\infty} \int_{0}^{2 \pi} \int_{0}^{\infty}\left(\left|\partial_{r} u\right|^{2}+4 r^{2}\left|\partial_{t} u\right|^{2}\right) r d r d \varphi d t \\
& +\beta^{2} \int_{-\infty}^{\infty} \int_{0}^{2 \pi} \int_{0}^{\infty} \frac{r^{2}}{r^{4}+t^{2}}|u|^{2} r d r d \varphi d t
\end{aligned}
$$

Now we apply Proposition 2 to the first integral on the right-hand side, completing the proof.

\section{REFERENCES}

[1] M. Sh. Birman, On the spectrum of singular boundary value problems, Mat. Sb. (N. S.) 55 (1961), no. 2, 125-174; English transl., Eleven Papers on Analysis, Amer. Math. Soc. Transl. (2), vol. 53, Amer. Math. Soc., Providence, RI, 1966, pp. 23-60. MR0142896 (26:463)

[2] L. D'Ambrosio, Some Hardy inequalities on the Heisenberg group, Differ. Uravn. 40 (2004), no. 4, 509-521; English transl., Differ. Equ. 40 (2004), no. 4, 552-564. MR2153649 (2007d:26016)

[3] _ Hardy inequalities related to Grushin type operators, Proc. Amer. Math. Soc. 132 (2004), no. 3, 725-734. MR2019949 (2005c:35050)

[4] E. B. Davies, A review of Hardy inequalities, The Maz'ya Anniversary Collection. Vol. 2 (Rostock, 1998), Oper. Theory Adv. Appl., vol. 110, Birkhäuser-Verlag, Basel, 1999, pp. 55-67. MR1747888 (2001f:35166)

[5] J. Dou, Q. Guo, and P. Niu, Hardy inequalities with remainder terms for the generalized BaouendiGrushin vector fields, Math. Inequal. Appl. 13 (2010), no. 3, 555-570. MR2662838 (2011e:35250)

[6] N. Garofalo, Unique continuation for a class of elliptic operators which degenerate on a manifold of arbitrary codimension, J. Differential Equations 104 (1993), no. 1, 117-146. MR.1224123(94i:35037)

[7] N. Garofallo and E. Lanconelli, Frequency functions on Heisenberg group, the uncertainty principle and unique continuation, Ann. Inst. Fourier (Grenoble) 40 (1990), no. 2, 313-356. MR1070830 (91i:22014)

[8] V. V. Grushin, A certain class of hypoelliptic operators, Mat. Sb. (N. S.) 83 (1970), no. 3, 456-473; English transl., Math. USSR-Sb. 12 (1970), 458-476. MR0279436 (43:5158)

[9] I. Kombe, Hardy, Rellich and uncertainty principle inequalities on Carnot groups, Preprint arXiv: math/0611850.

[10] A. Laptev and T. Weidl, Hardy inequalities for magnetic Dirichlet forms, Mathematical Results in Quantum Mechanics (Prague, 1998), Oper. Theory Adv. Appl., vol. 108, Birkhäuser, Basel, 1999, pp. 299-305. MR1708811(2001d:35146)

[11] V. G. Maz'ya, Sobolev spaces, Leningrad. Univ., Leningrad, 1985; English transl., Springer-Verlag, Berlin, 1985. MR0807364 (87g:46055) MR0817985 (87g:46056)

[12] P. Niu, Y. Chen, and Y. Han, Some Hardy-type inequalities for the generalized Baouendi-Grushin operators, Glasg. Math. J. 46 (2004), no. 3, 515-527. MR.2094807 (2006a:35040)

[13] E. M. Stein, Harmonic analysis: real-variable methods, orthogonality, and oscillatory integrals, Princeton Math. Ser., vol. 43, Princeton Univ. Press, Princeton, NJ, 1993. MR1232192 (95c:42002)

Stockholm University, SE-106 91 Stockholm, Sweden

E-mail address: lior@math.su.se

Imperial College London, 180 Queen's Gate, London SW7 2AZ, United Kingdom

E-mail address: a.laptev@imperial.ac.uk

Received 28/NOV/2010

Translated by THE AUTHORS 